\title{
LAS TECNOLOGÍAS DE LA INFORMACIÓN Y LA COMUNICACIÓN EN LA UNIVERSIDAD COLOMBIANA: EVOLUCIÓN Y PROSPECTIVA
}

\author{
Fredy Yesid Mesa Jiménez ${ }^{1}$ \\ Universidad Pedagógica y Tecnológica de Colombia - Colombia \\ Grupo de Investigación HISULA \\ fredy.mesa@uptc.edu.co
}

Recepción: 25/07/2011

Evaluación: 29/05/2012

Aceptación: 15/11/2012

Artículo de Revisión

doi: http://dx.doi.org/10.9757/Rhela.19.03

\section{RESUMEN}

La presente revisión sintetiza la memoria histórica de la evolución y prospectiva de las Tecnologías de la Información y la Comunicación (TIC) en la universidad colombiana, con el fin de realizar un acercamiento a la comprensión del estado actual y un aporte conceptual que oriente la toma de decisiones, las políticas y los programas en TIC hacia sus tendencias en el ámbito universitario.

Las TIC son sinónimo de modernización, calidad, productividad, mejores servicios y apoyo a los procesos educativos, por ende, algunas universidades intentan ir a su ritmo, ya que consideran que las pone en una situación ventajosa, por lo que es necesario indagar cómo se afrontó este proceso. Esta síntesis siguió la metodología de la Historia de la educación ${ }^{2}$. Las TIC en la universidad colombiana se integraron principalmente en los ejes de docencia y administración mediante la autonomía universitaria, avanzando en cada contexto hacia una e-universidad. Hoy se han materializado algunas de las potencialidades y servicios, se cuenta con políticas públicas y está en crecimiento la modalidad virtual, pero se debe avanzar hacia la tendencia Universidad 2.0.

Palabras clave: Revista Historia de la educación latinoamericana, tecnología de la información, tendencia educacional, enseñanza superior.

\footnotetext{
1 Candidato a Doctor en Ciencias de la Educación de RUDECOLOMBIA. Docente asistente de la Universidad Pedagógica y Tecnológica de Colombia, integrante del grupo de investigación Historia y Prospectiva de la Universidad Latinoamericana - HISULA - UPTC.

2 "Es una rama de la historia y de la cultura, que tiene por objeto el estudio y el conocimiento de la teoría o el hecho educativo en el tiempo y en el espacio en que tuvieron lugar”. Ávila Fernández, Alejandro, Calderón España, María Consolación, Cortés Giner, María Isabel, Montero Pedrera, Ana María. Historia de la Educación, Cuestiones previas y perspectivas actuales. (Sevilla: GI.P.E.S., 1996), 60.
} 


\section{INFORMATION AND COMMUNICATION TECHNOLOGIES IN COLOMBIAN UNIVERSITIES: EVOLUTION AND FUTUROLOGY}

\section{ABSTRACT}

The current review summarizes the historic memory of the evolution and futurology concerning the Information and Communication Technologies (ICT) in Colombian Universities. This is in order to make an approach to understand the current state and provide a conceptual input that advices decision making, policies and programs regarding ICTs towards its trends in universities.

ICTs mean modernization, quality, productivity, better services and support to educational processes. Therefore, some universities try to be up-to-date because they consider that ICTs gives them the advantage, which is why it is imperative to investigate how this process was faced. This synthesis followed the methodology of the History of Education ${ }^{3}$. ICTs were integrated in the Colombian universities mainly in the concepts of teaching and management through university's autonomy, advancing in every context towards an on-line university. Nowadays, the potential and services have become real, there are public policies available and on-line modality is increasing, however the University 2.0 trend must be reached.

Key words: History of Latin American Education Journal, information and communication technologies, educational trend, bigher education.

\section{AS TECNOLOGIAS DA INFORMAÇÃO E DA COMUNICAÇÃO NA UNIVERSIDADE COLOMBIANA: EVOLUÇÃO E PROSPECTIVA}

\section{RESUMO}

A presente revisão sintetiza a memória histórica da evolução e prospectiva das Tecnologias da Informação e da Comunicação (TIC) na universidade colombiana, com a finalidade de permitir uma compreensão do estado atual e uma contribuição conceitual que oriente a tomada de decisões, as políticas e os programas em TIC acerca de suas tendências no âmbito universitário.

AS TIC são sinónimos de modernização, qualidade, produtividade, melhores serviços e apoio aos processos educativos, e por causa disso algumas universidades objetivaram desenvolvê-las em seu próprio ritmo, já que consideram que as coloca em uma situação vantajosa, pelo que se faz necessário como se afrontou este processo.

Esta síntese seguiu a metodologia da História da Educação. As TIC na universidade colombiana se integraram principalmente nos eixos da docência e da administração, mediante a autonomia universitária, avançando em cada contexto de uma universidade. Hoje se materializaram algumas das potencialidades e serviços, se conta com políticas públicas e está em crescimento a modalidade virtual, porém de deve avançar para a tendência de Universidade 2.0.

Palavras-chave: Revista História da educação latinoamericana, tecnologia da informação, tendência educacional, ensino superior.

\section{INTRODUCCIÓN}

\section{La presente revisión sintetiza la memoria histórica de la evolución} y prospectiva de las Tecnologías de la Información y la Comunicación (TIC) en la universidad colombiana comprendida entre1957 y 2010, con el fin de realizar un acercamiento a la comprensión del estado actual y un aporte conceptual que oriente la toma de decisiones, las políticas y los

\footnotetext{
"It is a branch of the history and culture that whose objective is the study and knowledge of educational facts in the time and place of their appearance". Ávila Fernández, Alejandro, Calderón España, María Consolación, Corts Giner, María Isabel, Montero Pedrera, Ana María. Historia de la Educación, Cuestiones previas y perspectivas actuales. (Sevilla, GI.P.E.S., 1996), 60.
} 
Las tecnologías de la información y la comunicación en la universidad colombiana: evolución y prospectiva

programas en TIC hacia las nuevas tendencias que conllevan estas tecnologías en el ámbito universitario.

La universidad, es una institución de trayectoria histórica que juega un papel transformador de la sociedad, por lo que indagar cómo se enfrentó el reto y las oportunidades que ofrecen las TIC a todos sus ámbitos, se convierte en un tema de vigencia.

Integrar las TIC a la educación universitaria ha sido un proceso complejo, a pesar de las acciones de fortalecimiento de los últimos años (algunas influidas por los procesos de registro calificado y acreditación), con el propósito de optimizar los recursos, apoyar, mejorar e innovar los procesos educativos para transformar la formación universitaria presencial, que ha estado ligada a un paradigma tradicional magistral, por tanto, surgen los siguientes interrogantes en el contexto colombiano: ¿Cómo se afrontó en Colombia el proceso de integración de las TIC en las universidades? ¿Cuáles son las nuevas tendencias de la implementación de las tecnologías en la universidad?

Este estudio siguió los pasos de la metodología Historia de la Educación, a través de la localización y recolección de fuentes primarias y secundarias (medios impresos y publicados en la Web), el diseño y aplicación de una encuesta dirigida al Departamento Nacional de Planeación, para examinar el seguimiento de las políticas nacionales en TIC. Finalmente, se organizó, categorizó e interpretaron las fuentes para realizar una síntesis explicativa que estableció conclusiones.

La reconstrucción del recorrido histórico - educativo de las TIC en la universidad colombiana, se remitió desde la llegada de la computación e Internet al país, el crecimiento de la informática en las universidades, el desarrollo de la educación virtual, los estudios, políticas y programas liderados por el Ministerio de Educación Nacional, la modernización del sector educativo, la e-universidad $^{4}$ y la prospectiva de las TIC en la universidad.

Entendida como "la aplicación intensiva, extensiva y estratégica de las nuevas tecnologías de la información, las telecomunicaciones e Internet (TIC) a todas las actividades de una universidad". Susana, Finquelievich y Alejandro Prince. Universidades y TICs en Argentina. Las universidades argentinas en la sociedad del conocimiento (2005), http://www.scribd.com/doc/4940962/Finquelievich-y-PrinceLAS-UNIVERSIDADES-ARGENTINAS-EN-LA (20 de noviembre de 2011). 


\section{Inicios de la computación moderna en el mundo y su llegada a Colombia}

La computación moderna surgió a finales de la década de los treinta y en los cuarenta, etapa en la que se llevaron a cabo proyectos de investigación ${ }^{5}$ ligados a universidades que demostraron la factibilidad de los computadores electrónicos ${ }^{6}$. Para ese entonces "parecía poco probable que muchos de estos voluminosos, incómodos y temperamentales monstruos se llegaran a construir fuera de las universidades, las instalaciones militares y los laboratorios estatales"’.

A finales de los años cincuenta, estas máquinas habían ganado la confianza entre las compañías y avanzaban gracias a la carrera espacial. Fue una época con exceso de optimismo en cuanto a las posibilidades que ofrecían los computadores ${ }^{8}$. Aquí brota la historia de la computación latinoamericana. En Colombia, la experiencia de las universidades fue consecuencia de acciones del sector productivo, ya que en 1957 llega el primer computador, un mainframe IBM $650^{\circ}$ adquirido por la empresa Bavaria; ese mismo año Coltejer adquirió una y las Empresas Públicas de Medellín y Ecopetrol lo hicieron en $1958^{10}$.

En otros países los primeros computadores fueron traídos por universidades, en México en 1958 se instala una IBM 650, en la Universidad Nacional Autónoma de México (UNAM), cuyo primer dueño fue la Universidad de California en los Ángeles (UCLA) ${ }^{11}$ y en Argentina la Facultad de Ciencias Exactas de la Universidad de Buenos Aires, adquirió una

5 En 1939, en la actual Iowa State University (EE.UU.), se crea la primera computadora electrónica digital de propósito especial, el ABC (Atanasoff-Berry Computer) y posteriormente en la Universidad de Pensilvania (EE. UU.) se originó el ENIAC (Computador e Integrador Numérico Electrónico), el primer computador electrónico de uso general terminada en 1945. Según Carlos Coello Coello, Breve historia de la computación y sus pioneros (México: Fondo de Cultura Económica, 2003), 160 y 318.

6 La aplicación de la electrónica para el procesamiento de la información evolución de tubos al vacío, a transistores (1947), circuitos integrados (1958) y microprocesadores (1971).

7 Tom Forester, Sociedad de alta tecnologia: la historia de la revolución de la tecnología de la información (México: Siglo Veintiuno editores, 1992), 33.

8 Senén Barro Ameneiro y Alberto Bugarín Diz, Fronteras de la computación (España: Ediciones Díaz de Santos, 2002), 19.

9 Computador a escala industrial fabricado en 1953 por la International Business Machines (IBM).

10 Álvaro Montes, "Llegaron los computadores", en 50 días que cambiaron la historia de Colombia de Revista Semana (Bogotá: Editorial Planeta, 2004), 237-238.

11 Daniel Ortiz Arroyo, Francisco Rodríguez Henríquez y Carlos Coello Coello, "Computadoras mexicanas: una breve reseńa técnica e histórica”, Revista Digital Universitaria Universidad Nacional Autónoma de México, vol. 9 , $\mathrm{N}^{\circ} 9$ (2008), 5 . 
computadora Mercury de la firma Ferranti instalada en 1961'12, conocida como Clementina, según Sadosky (citado por Correa) "porque modulando un pitillo que emitía la máquina, se escuchaba Clementine, una canción inglesa muy popular"'13.

Los dos primeros IBM 650 que llegaron al país, "fueron a parar a las Universidades Nacional y Los Andes"14, específicamente a esta última en 1963 al Departamento de Ingeniería Eléctrica ${ }^{15}$ y fue, en estas instituciones, donde se crearon los primeros programas en esta nueva disciplina. En el segundo semestre de 1966 la Universidad Nacional crea la Maestría en Sistemas, primera de Latinoamérica en este nivel ${ }^{16}$ y en 1967 , se funda la carrera de Ingeniería de Sistemas en la Universidad de los Andes ${ }^{17}$.

\section{Evolución de la informática en las Universidades Colombianas}

En 1966 y 1967 en la Universidad Nacional y en la Universidad Industrial de Santander, se instalaron dos computadores IBM/1620 ${ }^{18}$, los cuales eran basados en transistores, a pesar de que a mediados de los sesenta aparecen las computadoras de tercera generación, a base de circuitos integrados. Por esta época los computadores eran escasos en el país ${ }^{19}$. En lo relacionado con la generación de tecnologías computacionales $^{20}$ en Colombia, se destaca el Computador Digital Didáctico (CODIDAC), realizado en la Pontificia Universidad Javeriana entre noviembre 1969 a febrero de $1971^{21}$, el cual era pionero para la época.

12 Pablo Jacovkis. Breve resumen de la historia de la computación en Argentina" (2004), http://www.sadio.org. ar $/$ modules.php?op=modload $\&$ name $=$ News $\&$ file $=$ article $\&$ sid $=50(29$ de enero, 2010)

13 Leonardo Correa, La llamaron Clementina (2005), http://edant.clarin.com/suplementos/informatica/ 2005/08/17/f-00511.htm (17 de septiembre, 2010).

14 Montes, "Llegaron los computadores", 237.

15 Oscar Guarín, "ACIS, Memorias de la Informática en Colombia", Revista Sistemas. Memoria informática de Colombia Edición $N^{\circ}$ 100. Asociación Colombiana de Ingenieros de Sistemas (2007), http://www.acis.org.co/ fileadmin/Revista_100/ED_100_INVESTIGACION.pdf (27 de octubre, 2012).

16 Oscar Guarín, "ACIS, Memorias de la Informática en Colombia".

17 Universidad de los Andes. Sitio Web del Departamento de Ingeniería de Sistemas y Computación, http:// sistemas.uniandes.edu.co/sitio/index.php?option=com_content\&view=article\&id=65\&Itemid=90 (17 de septiembre, 2010).

18 Departamento Administrativo Nacional de Estadística, Primer censo nacional de recursos informáticos en el sector público (Colombia: Editor DANE, 1985), 7.

19 Luis Uribe, "CODIDAC", Revista Tekhne de la Facultad de Ingeniería No 7. Universidad Católica Andrés Bello (2004): 50.

20 Se resaltan las universidades públicas mexicanas, que a finales de los 70 y mediados de los 80 diseńaron variedad de sistemas de cómputo basados en microprocesadores.

21 Luis Uribe, "Proyectos realizados", http://www.scribd.com/doc/20253455/UribeUSBProy1d-Publicar (15 de octubre, 2012). 
En los años setenta los computadores se volvían menos voluminosos, más seguros, económicos y veloces debido a los adelantos en electrónica ${ }^{22}$ y aparecen los sistemas de cuarta generación, basados en microprocesador. La disciplina se hallaba en expansión en el país y crecía el interés al interior de las universidades, por lo cual empiezan a crear centros de cómputo, que en el caso de la UPTC fue en 1972, con el fin de sistematizar labores administrativas y académicas ${ }^{23}$. Se crea la Asociación Colombiana de Usuarios de Computadores (ACUC) en 1972 y la Asociación Colombiana de Ingenieros de Sistemas (ACIS) en $1975^{24}$.

Desde este momento, las universidades afrontaron por su cuenta el reto de introducir la tecnología en todos sus ámbitos, mediante la autonomía universitaria, lo que influyó para que la integración de las TIC y la virtualización, no se diera del mismo modo en todas las universidades, hasta 2008, cuando se publicó los "Lineamientos para la formulación de planes estratégicos de incorporación de TIC en Instituciones de Educación Superior (IES)" 25 .

Los eventos para finales de los ochenta y en los noventa, señalados por Maldonado fueron los foros de la Asociación Colombiana de Universidades (ASCUN) sobre informática en la educación universitaria y el primer Simposio colombiano de informática, educación y capacitación; en 1989 Colciencias, y la Universidad de los Andes crean una revista en informática educativa $^{26}$. Se destacan Compuexpo 90, en el que se presentó el Museo Colombiano de Informática ${ }^{27}$, conferencias y seminarios respaldados por universidades $^{28}$. En 1990 nace la Red Iberoamericana de Informática Educativa (RIBIE) cuyo nodo en Colombia es RIBIECOL ${ }^{29}$, siendo la organi-

22 Los avances tecnológicos en computación y su adopción transversal a cualquier sector, continúan gracias a la aplicación de inteligencia artificial, nanotecnología, robótica, sistemas expertos, entre otras tendencias.

23 Universidad Pedagógica y Tecnológica de Colombia. Resolución Rectoral Número 414 de 1972.

24 Oscar Guarín, ACIS, Memorias de la Informática en Colombia.

25 Desarrollado por el MEN en convenio con el grupo LIDIE de la Universidad de los Andes.

26 Luis Facundo Maldonado Granados y Paola Maldonado Rey, "Nuevas tecnologías aplicadas a la educación. Estado del arte de la investigación, 1990 - 1999”, en Estados del Arte de la Investigación en Educación y Pedagogía en Colombia, eds. Myrian Henao Willes y Jorge Orlando Castro (Bogotá: ICFES, 2001), 146-147.

27 El Tiempo, "Museo de Informática", Periódico El Tiempo, Bogotá, 8 de agosto de 1990. http://www.eltiempo. com/archivo/documento/MAM-69828 (25 de febrero, 2013).

28 Jaime Mejía Mazuera, "Para qué sirven los computadores", Periódico El Tiempo, Bogotá, agosto 8 de 1990 , http://www.eltiempo.com/archivo/documento/MAM-69998 (25 de febrero, 2013).

29 Desde 1992 organiza cada dos años el Congreso de Informática Educativa. 
zación más antigua e importante en este tema en el país ${ }^{30}$ y se crean grupos de investigación en la relación educación e informática ${ }^{31}$. Estos hechos evidencian la propagación en investigación, actualización y generalización de la informática en el país.

La década de los noventa se identifica por rápidos avances tecnológicos y porque los computadores llegan a un mayor número de personas y organizaciones, crece la comunidad investigativa y se efectúa la conexión a Internet en Colombia. Asimismo, inicia la educación a distancia/virtual, ya que en 1992 "el Instituto Tecnológico y de Estudios Superiores de Monterrey en convenio con la Universidad Autónoma de Bucaramanga \{... of ofecian programas académicos a distancia (maestrías), mediante clases satelitales producidas en México", y posteriormente (1995/1996) se vincularon a este convenio universidades adscritas a la Red Universitaria José Celestino Mutis ${ }^{32}$.

La Ley 115 de 1994 estableció la Tecnología e Informática en el nivel de básica, lo que favoreció la alfabetización informática, a pesar de falencias en infraestructura existente en ese momento para esas instituciones. Esto desencadena la creación de Licenciaturas en Informática ${ }^{33}$, cuyos egresados de forma gradual se han vinculado al sistema educativo nacional.

\section{Internet en Colombia}

Según Meneses, en 1986 las Universidades de los Andes, Nacional y del Norte iniciaron pruebas para una conexión a la red internacional, pero el proyecto no se desarrolló completamente. En 1990 las principales univer-

30 Luis Facundo Maldonado Granados, "RIBIECOL - 20 años de vida" (Memorias Congreso Internacional de Informática Educativa. Aulas virtuales, diseño pedagógico, simulaciones y cibercultura, Popayán 14, 15 y 16 de julio de 2010, CD-ROM. RIBIECOL, 2010).

31 El Laboratorio de Investigación y Desarrollo sobre Informática en Educación (LIDIE) de la Universidad de Los Andes, concebido desde 1985 inicialmente como Grupo de Informática Educativa - GIE y el grupo Didáctica y Nuevas Tecnologías, de la Universidad de Antioquia cuyo origen se remonta a 1986.

32 Facundo Ángel, "La Educación Superior Virtual en Colombia", en La educación superior virtual en América Latina y el Caribe, ANUIES-UNESCO (México: ANUIES, 2004), 193.

33 Se destacan la Licenciatura en Informática Educativa de la UPTC (Acuerdo No. 022 de 1994 del Consejo Superior Universitario), Licenciatura en Educación básica con énfasis en Tecnología e Informática en la Corporación Universitaria Minuto de Dios (Acuerdo del Consejo Superior No 087 del 25 de agosto de 1999), Licenciatura en Educación Básica con Énfasis en Informática de la Universidad del Magdalena (acreditación previa según Resolución 3306 del 7 de diciembre de 2000), Licenciatura en Informática y Medios Audiovisuales de la Universidad de Córdoba (Registro calificado Resolución 3311 de diciembre 7 de 2000), Licenciatura en Informática de la Universidad de Nariño y la Licenciatura en Comunicación e Informática Educativa de la Universidad Tecnológica de Pereira. 
sidades del país con el apoyo del Instituto Colombiano para el Fomento de la Educación Superior (ICFES), crearon la Red Universitaria Colombiana (RUNCOL) que funcionó a través de la Universidad de los Andes, que contaba con la infraestructura para operar con la red Bitnet. Sin embargo, su acceso era limitado y no se podían usar en línea los servicios que esta red ofrecía ${ }^{34}$.

Según la Revista de Ingeniería de la Universidad de los Andes, en 1991, el cambio a Bitnet II, requirió que esta solicitara el manejo de las asignaciones de direcciones y administración del dominio ".co", trámite que fue aprobado $^{35}$. En 1993 y gracias al backbone nacional, conformado por la EAFIT, la Universidad del Valle y Los Andes, se empezó a desarrollar de manera local los servicios de Internet. En diciembre de 1993, delegados del ICFES, Colciencias, EAFIT, Universidad del Valle y Los Andes acuerdan la creación de la corporación InterRed para administrar la conexión a Internet, lo cual se concreta hasta 1996. Como la Universidad de los Andes tenía la infraestructura técnica y la experiencia, se encargó de montar y poner en marcha la administración del primer proveedor de servicios de Internet (ISP), siendo la fecha de entrada de Internet a Colombia el 4 de junio de $1994^{36}$.

A finales de 1995, algunas universidades ${ }^{37}$ ya contaban con páginas en la Web $^{38}$. Según Valencia ${ }^{39}$, desde la introducción del Internet, hasta los primeros meses de 1997, la mayoría de los usuarios estaban en las

34 Arnaldo Meneses, El sector de las telecomunicaciones en Colombia en la década de los 90 's. (Comisión de Regulación de Comunicaciones de Colombia, 2000), 153. http://www.crcom.gov.co/images/stories/crtdocuments/BibliotecaVirtual/publi_sector90/LibroTelec.pdf (15 de junio, 2010).

35 Las organizaciones que desearan registrar un dominio "co" debían hacerlo a través de la Universidad de los Andes. Este servicio se prestó durante 18 años hasta el 7 de febrero de 2010, http://www.uniandes.edu.co/ la_universidad/Informatica.php.

36 Fernando, Salcedo, "Desde la academia, Internet para Colombia". Revista de Ingeniería Universidad de los Andes $\mathrm{N}^{\circ} 23$ (2006), 146-148.

37 Se destacan las Universidades: Los Andes, Nacional, del Cauca, del Valle, de Antioquia, EAFIT, Javeriana, Antonio Nariño y la Corporación Universitaria de Ibagué.

38 Carlos Manjarrés, "Presencia de Colombia en Internet", Periódico El Tiempo, Bogotá, noviembre 20, 1995.

39 Destaca en su estudio de 1998 al interior de la Universidad Javeriana, el uso de Internet para la comunicación entre compañeros, amigos, familiares y como herramienta informativa, no tanto académica, como si de entretenimiento. Algunos usuarios aprendían a utilizarlo por cuenta propia y apoyados por sus amigos. Las dificultades técnicas apartaban a muchas personas de los computadores y de Internet y el conocimiento de otros idiomas dificultaba su interacción con personas de otros países y las búsquedas de información. 

universidad colombiana: evolución y prospectiva

universidades de las principales ciudades del país ${ }^{40}$. A finales de 1999 el $77 \%$ del uso de Internet, se concentró en las grandes empresas, las universidades y órganos estatales ${ }^{41}$. Lo anterior, muestra a las universidades como actores fundamentales de la conexión a Internet y su difusión.

\section{La Educación Virtual en Colombia}

El año de 1998, de acuerdo con Ángel, podría considerarse como el año de inicio de la virtualidad en Colombia, dado que dos instituciones ${ }^{42}$ comienzan a ofrecer programas de pregrado soportados en tecnologías virtuales ${ }^{43}$. Para 2002, según un análisis de Zapata a una muestra de las universidades sobre experiencias educativas colombianas apoyadas en Internet, era "clara la tendencia en las instituciones de educación superior a trabajar en forma aislada y son muy pocas las alianzas que hacen entre ellas, algunas cuantas tienen convenios con universidades extranjeras para ofrecer programas académicos" ${ }^{\prime 4}$.

Posteriormente, crecen las universidades presenciales y a distancia con campus virtual, como política de apoyo a los procesos educativos, para lo que crean dependencias con profesionales interdisciplinarios (programadores, diseñadores gráficos, pedagogos, entre otros) para liderar proyectos de educación virtual. Algunos casos de comités y dependencias son:

40 Juan Carlos Valencia Rincón, "Internet en Colombia. Alucinación, información y comunicación” (tesis de maestría en Comunicación, Pontificia Universidad Javeriana. Facultad de Comunicación y Lenguaje, 1998) http://www.geocities.com/BourbonStreet/Quarter/7014/Tesis.doc (26 de mayo de 2009).

41 Meneses, "El sector de las telecomunicaciones", 156.

42 Según Facundo Ángel, uno en la Universidad Militar Nueva Granada (institución oficial) y tres en la Fundación Universitaria Católica del Norte (institución privada), primera universidad totalmente virtual del país. Además la Universidad Nacional inicia el desarrollo de cursos en línea y la Universidad de los Andes el proyecto piloto denominado Sistema Interactivo de Cursos Universidad de los Andes (SICUA), espacio para que docentes y estudiantes compartieran información, accedieran a la programación y al contenido de los cursos.

43 Ángel, "La Educación Superior Virtual en Colombia", 193-194.

44 Donna Zapata Zapata, Contextualización de la enseñanza virtual en la educación superior. (Bogotá: ICFES, 2002), http://blade1.uniquindio.edu.co/uniquindio/ntic/lineamientos/nuevos\%20libros/arc_88.pdf (21 de mayo, 2012). 


\section{Tabla 1. Algunos comités y dependencias de Educación Virtual (2003 - 2004)}

\begin{tabular}{|c|c|c|}
\hline Universidad & Nombre del comité o dependencia & Año de creación \\
\hline Pontificia Universidad Javeriana & $\begin{array}{c}\text { Centro de Educación Asistida por Nuevas } \\
\text { Tecnologías (CEANTIC) }{ }^{45}\end{array}$ & 2003 \\
\hline $\begin{array}{l}\text { Universidad Pedagógica y Tecno- } \\
\text { lógica de Colombia (UPTC) }\end{array}$ & Comité asesor de la Educación Virtual ${ }^{46}$ & 2003 \\
\hline $\begin{array}{l}\text { Universidad Tecnológica de } \\
\text { Pereira (UTP) }\end{array}$ & Univirtual $^{47}$ & 2004 \\
\hline $\begin{array}{l}\text { Universidad Nacional de } \\
\text { Colombia (UNAL) }\end{array}$ & $\begin{array}{c}\text { Inicialmente Programa Universidad Virtual } \\
\text { (1999-2004) Dirección Nacional de Servicios } \\
\text { Académicos Virtuales }{ }^{48} \text {. }\end{array}$ & 2004 \\
\hline
\end{tabular}

Fuente: compilado por el autor.

Las acciones de estas dependencias influyeron al interior de las instituciones. Son diferentes en infraestructura, políticas, estímulos, alianzas, seguimiento de las experiencias y respaldo institucional, junto a la visión de ofrecer programas totalmente virtuales. Con su implementación cambia el modelo pedagógico en cada contexto y con diferentes resultados. En modalidad presencial, el apoyo virtual es un valor agregado y algunas universidades cuentan con mecanismos que velan por su calidad.

Según el proyecto europeo EQUIBELT ${ }^{49}$, existe preocupación ya que "esta integración de la tecnología no está suponiendo un proceso de innovación, sino que se está anexando a situaciones de aprendizaje tradicionales" ${ }^{50}$, pero se debe considerar que si se consolidan procesos y materiales de calidad, se afectan positivamente los procesos educativos en flexibilidad de tiempos, espacios y modelos de enseñanza, por lo que no sería lo mismo.

45 Pontificia Universidad Javeriana, Informe del Rector al Consejo de Regentes 2003 (Bogotá: Editorial Pontificia Universidad Javeriana, 2003), 30.

46 Universidad Pedagógica y Tecnológica de Colombia. Resolución Rectoral Número 2848 de 2003.

47 Martha Isabel Tobón Lindo, et al., La formación docente al incorporar las TIC en los procesos de enseńanza y aprendizaje. Una propuesta para la Universidad Tecnológica de Pereira (2010), http://www.slideshare.net/ misabell/el-docente-y-las-tic-4476500 (31 de agosto, 2010).

48 Universidad Nacional de Colombia, Acuerdo Número 034 de 2004 del Consejo Superior Universitario (Bogotá: Universidad Nacional, 2004), http://www.unal.edu.co/secretaria/normas/csu/2004/A0034_04S.pdf (25 de febrero, 2013).

49 Education Quality Improvement by E-learning Technology.

50 Citado por Carlos Castańo Garrido, et al., "Elearning 2.0", en Nuevos escenarios pedagógicos para el aprendizaje digital (Universidad del País Vasco, 2009), http://ocw.ehu.es/ciencias-sociales-y-juridicas/nuevos-escenarios-pedagogicos-para-el-aprendizaje-digital/bloque-3-estrategias-de-aprendizaje-2-0-para-la-innovacion-pedagogica/ tema-1-elearning-2.0 (2 de julio, 2010). 
Actualmente, algunas universidades ${ }^{51}$ cuentan con programas de pregrado en modalidad virtual y/o distancia-virtual ${ }^{52}$. Los cursos de educación continuada y posgrados con apoyo de TIC son extensos. La tendencia no es pasajera ya que permite expandir la oferta educativa, lo que es acorde con el MEN, que para mejorar sus indicadores de cobertura, desde 2003 fomentó la oferta virtual y en 2008, por medio de la Asociación Elearning 2.0 Colombia, comenzó a transformar 18 programas a distancia técnicos profesionales y tecnológicos a programas virtuales ${ }^{53}$, estrategia también del Plan Nacional de TIC 2008-2019.

En junio de 2010, el MEN lanzó una campaña de educación superior virtual, entendida como "una alternativa de formación que permite a las personas realizar su proceso educativo en un lugar distinto al salón de clases: el ciberespacio" "54, que en ese momento, contaba "con 147 programas, con más del 80 por ciento de virtualidad"55. Este apoyo formal, pretendió aumentar la acogida en esta modalidad educativa y superar el debate acerca de su calidad.

\section{Estudios del Ministerio de Educación Nacional}

El estudio Modelos virtuales en las instituciones de IES colombianas ${ }^{56}$, que inició a finales de 2005, identificó cuatro tipos de instituciones según el uso de las TIC: vanguardistas, $13 \%$; cooperadores, 13\%; autosuficientes 37\% y escépticas, $37 \%$ " último grupo la mayoría del sector público" 58 .

51 Se destacan la Fundación Universitaria Católica del Norte, la Universidad Autónoma de Bucaramanga, Fundación Universitaria CEIPA, la Pontificia Universidad Javeriana, la Universidad Cooperativa de Colombia y el Politécnico Grancolombiano.

52 En la literatura no se encontró consenso con respecto a su definición. Se puede decir que es un tipo de educación a distancia que combina encuentros presenciales y el apoyo de las TIC en los procesos de aprendizaje.

53 Ministerio de Educación Nacional, "Mineducación lanzó campaña de educación superior virtual como una alternativa real”. (2010). http://www.mineducacion.gov.co/cvn/1665/w3-article-237475.html (22 de noviembre de 2011).

54 Colombia Aprende. “¿Qué es la educación virtual?”, (s.f.). http://www.colombiaaprende.edu.co/html/productos/1685/article-229097.html (22 de abril, 2012).

55 Ministerio de Educación Nacional. Balance de la Revolución Educativa 2002 - 2010 (2010), http://www.mineducacion.gov.co/1621/articles-231469_archivo_pdf_discurso.pdf (08 de junio, 2010).

56 Se aplicó a 171 instituciones entre públicas y privadas (técnicas y tecnológicas, instituciones universitarias y universidades), teniendo en cuenta aspectos como: organización institucional, metodologías de aprendizaje y trabajo colaborativo en red con otras IES nacionales e internacionales.

57 Ministerio de Educación Nacional. Las tecnologias, aliadas estratégicas de la educación superior (2006?), http:// www.mineducacion.gov.co/cvn/1665/fo-article-103872.pdf (19 de abril, 2012).

58 Carolina Botero, Seminario del Ministerio de Educación en Colombia Estrategias para el Uso de Objetos de Aprendizaje y Redes de Alta Velocidad (2006), http://www.karisma.org.co/carobotero/index. 
Otro estudio fue el Diseño de lineamientos para la formulación de planes estratégicos de incorporación de TIC en IES colombianas, que en 2008 realizó un proceso piloto que acompañó a 28 instituciones y que se expandió en 2009 a $64 \mathrm{IES}^{59}$. Algunos países crearon sistemas para revisar anualmente el avance de las TIC en las universidades, en Estados Unidos ${ }^{60}$ desde 1990 , en el Reino Unido ${ }^{61}$ a partir de 1996-7 y UNIVERSITIC ${ }^{62}$ en España desde 2006. Colombia aún no practica la revisión anual de este avance.

\section{Políticas y programas liderados por el Ministerio de Educación Nacional}

En Latinoamérica, las primeras políticas públicas sobre los temas de la informática, las telecomunicaciones o la computación, se encuentran en los años 1960-1970, especialmente en Brasil y México ${ }^{63}$. En Colombia, aparece en el año 2000, la primera política de Estado ${ }^{64}$ a largo plazo, denominada Agenda de conectividad: El Salto a Internet (CONPES 3072) y que según Rincón, se formuló con base en el programa desarrollado por el Gobierno de Canadá (Connecting Canadians), ajustado a la realidad social, económica y política colombiana ${ }^{65}$.

El objetivo del CONPES 3072 fue "impulsar el desarrollo socialy económico de Colombia mediante la masificación de las tecnologias de la información" "66, mirada determinista con respecto a la tecnología como promotor de cambios. Fue coordinado inicialmente por el programa presidencial para el desarrollo de

php/2006/07/27/seminario-del-ministerio-de-educacion-en-colombia-estrategias-para-el-uso-de-objetos-deaprendizaje-y-redes-de-alta-velocidad/ (19 de abril, 2012).

59 Colombia Aprende, Estrategia Planes TIC (Bogotá: Ministerio de Educación Nacional (s.f.), http://www.colombiaaprende.edu.co/html/directivos/1598/article-201182.html (20 de agosto, 2010).

60 Denominado "National Survey of Computing and Information Technology in American Higher Education".

61 Estudio "Higher Education Information Technology Statistic (HEITS).

${ }_{62}$ Analiza sobre los ejes estratégicos de: enseñanza-aprendizaje, investigación, gestión universitaria y de la información, formación, cultura y organización de las TIC.

63 Martin Hilbert, Sebastián Bustos, y João Carlos Ferraz, Estrategias nacionales para la sociedad de la información en América Latina y el Caribe (Naciones Unidas, 2005), 9.

64 Chile y Argentina son los primeros países en establecer decretos en 1998, Brasil en 1999, Colombia y Venezuela en el año 2000, según Hilbert, Bustos y Ferraz, "Estrategias nacionales", 28.

65 Erick Rincón Cárdenas, Manual de derecho de comercio electrónico y de Internet (Bogotá: Editorial Universidad del Rosario, 2006), 321.

66 Colombia. Consejo Nacional de Política Económica y Social. Departamento Nacional de Planeación. Ministerio de Comunicaciones. Documento CONPES 3072 de 9 de febrero de 2000, 12. 

universidad colombiana: evolución y prospectiva

las $\mathrm{TIC}^{67}$ pero al suprimir sus funciones, se asumieron por el Ministerio de Comunicaciones ${ }^{68}$; de acuerdo con Vanegas, este cambio fue debido al factor económico, ya que quien tenía el dinero era el fondo de comunicaciones ${ }^{69}$.

Los documentos CONPES establecen recomendaciones que surgen de acuerdos y en las que se responsabiliza a entidades del orden nacional, para articularlas a los planes nacionales de desarrollo. Así, el CONPES 3072 contiene los compromisos y los responsables, pero no estableció la duración e indicadores de seguimiento y coinciden, los entrevistados, que aún sigue vigente ${ }^{70}$. Fue elaborado por el Ministerio de Comunicaciones y el Departamento Nacional de Planeación ${ }^{71}$.

El CONPES 3072 no tuvo seguimiento de cumplimiento a través de SISCONPES ${ }^{72}$, ya que para ese entonces, todavía no se había implementado esta metodología ${ }^{73}$. A través del Sistema de Gestión y Seguimiento a las Metas de Gobierno ${ }^{74}$, algunos programas como RENATA, Computadores para educar y Gobierno en línea, han tenido registro de su avance.

En la última década los principales programas en TIC enfocados a educación superior, por parte del Ministerio de Educación Nacional (MEN) fueron: el Banco Nacional de Objetos de Aprendizaje, la Red Virtual de Tutores (RVT) y la Red Nacional de Tecnología Avanzada (RENATA), concretados entre 2006 y 2007, que generó, para ese entonces, un entorno propicio para su provecho por parte de las universidades ${ }^{75}$.

Actualmente las políticas, según el Plan Vive Digital' ${ }^{6}$, se orientan al crecimiento de la Educación Virtual, la expansión de RENATA y un Sis-

\footnotetext{
Colombia. Presidencia de la República. Decreto 127 de 2001.

Colombia. Presidencia de la República. Decreto 3107 de 2003.

Entrevista a Vanegas Murcia, Eliécer, Bogotá, 6 de octubre de 2011.

Entrevista a Vanegas Murcia, Eliécer, Bogotá, 6 de octubre de 2011.

Hilbert, Bustos, y Ferraz, "Estrategias nacionales", 50.

Es un sistema de seguimiento de los compromisos de los Documentos CONPES.

Entrevista a Barbosa, Adriana, Bogotá, 6 de octubre de 2011.

Véase: http://www.sigob.gov.co.

Fredy Yesid Mesa Jiménez y Ángela Marcela Soler Fonseca, "Políticas públicas en Tecnologías de la Información y la Comunicación y su incidencia en la universidad colombiana (2000-2008)”, en Memorias Congreso de Investigación y Pedagogía II nacional I internacional. Perspectivas retos y transformaciones en contextos educativos (Tunja: Maestría en Educación-UPTC, 2011), 270.

76 En el Plan Nacional de Desarrollo 2010-2014, el plan sectorial en TIC se denomina "Vive Digital".
} 
tema de Innovación Educativa ${ }^{77}$. Lo anterior se puede resumir en acciones sobre infraestructura, contenidos, cualificación y apropiación, temas recurrentes dentro de los lineamientos del MEN.

\section{Modernización tecnológica del sector educativo}

La Revolución educativa ${ }^{78}$ (2002-2010), se caracterizó por la continuación de políticas educativas neoliberales, que planteó mejorar la eficiencia del sector educativo, por lo que el $90 \%$ de los procesos del Ministerio de Educación se apoyan en sistemas de información, siendo líder entre las entidades del Estado en la política de Gobierno en Línea ${ }^{79}$.

La educación superior y el MEN, se articulan mediante los siguientes sistemas: SNIES ${ }^{80}$, que canaliza la información y estadísticas, el SPA$\mathrm{DIES}^{81}$, el cual "ayudó a generar acciones para reducir la tasa de deserción por cohorte que entre 2002 y 2009 pasó de 50\% a 45\%." ", el SACES ${ }^{83}$, que permite realizar trámites de registro calificado y un sistema de información que hace seguimiento a los egresados de la educación superior ${ }^{84}$.

Es generalizada la modernización tecnológica del sector educativo, influido en parte, porque las TIC son necesarias para cumplir con las funciones misionales, articular instituciones, asimismo, por la política de competitividad y de racionalización económica, por tanto, a pesar de su alto precio pocos son los que se oponen a su integración.

\section{La e-universidad en Colombia}

Las TIC en las universidades, en sus inicios, se enfocaron a optimizar labores administrativas ${ }^{85}$, hoy en día se han extendido a los ejes misionales

77 Ministerio de Tecnologías de la Información y las Comunicaciones, "Vive Digital Colombia. Documento Vivo del Plan. Versión 1.0”, (2011), 73-74.

78 Nombre fijado al sector educativo por el gobierno nacional en el Plan de Desarrollo Nacional 2002-2006.

79 Ministerio de Educación Nacional, Balance de la Revolución Educativa 2002-2010. http://www.mineducacion. gov.co/1621/articles-231469_archivo_pdf_discurso.pdf

80 Sistema de Información para la Educación Superior.

81 Sistema para la Prevención y Análisis de la Deserción en las Instituciones de Educación Superior.

82 Ministerio de Educación Nacional, Balance de la Revolución Educativa 2002-2010, http://www.mineducacion. gov.co/1621/articles-231469_archivo_pdf_discurso.pdf

83 Sistema de Aseguramiento de la Calidad de la Educación Superior.

84 Consejo Nacional de Acreditación. El sistema de mejoramiento continuo del Consejo Nacional de Acreditación (2009), http://www.cna.gov.co/1741/articles-186379_recurso_1.pdf (22 de noviembre, 2011).

85 El marcado uso administrativo, debe verse como favorable ya que ha obligado a una mayor alfabetización digital de la comunidad académica. 
de docencia, investigación y extensión ${ }^{86}$. Lo anterior se realiza a través de una planeación universitaria para desarrollar avances en TIC a nivel institucional. Algunas universidades tienen más integrados sus sistemas de información, pero en general lo vienen persiguiendo desde hace años.

Algunas universidades modificaron sus servicios ${ }^{87}$ para hacer uso de las TIC, influido por una visión de reducción de costos en publicidad, internacionalización y en personal al hacer más eficientes algunos procesos. Otro ejemplo de uso, fue reglamentar sesiones no presenciales del Consejo Superior Universitario de la Universidad Nacional de Colombia, para efectuarse por medios telefónicos o electrónicos cuando la urgencia de un tema lo amerite ${ }^{88}$.

En general, el uso extendido de servicios TIC, multimedia, Internet y campus virtuales han cambiado algunos procesos académico-administrativos y la forma en que se relacionan las personas y las instituciones educativas. Se puede resumir, que las TIC juegan un papel fundamental para cumplir con las funciones misionales de la universidad, en la sociedad de la información y del conocimiento.

\section{Futuro de las TIC en la Universidad}

El nuevo paradigma de la aplicación de las TIC en la universidad, es la Universidad 2.0, en donde la Web 2.0 y la actitud del usuario para hacer de esta un medio social, colaborativo, participativo, interactivo y de contenidos abiertos al servicio educativo ${ }^{89}$. Otras tecnologías son las pizarras digitales ${ }^{90}$, que se vienen incorporando en España, Reino Unido y a nivel latinoamericano. México desarrolla un proyecto para dotar 300.000 aulas $^{91}$;

86 Son ejemplo de ello el uso de intranet y sistemas de información para la administración de estudiantes, profesores, egresados, información bibliográfica, investigación, evaluación de profesores y sistemas contables y financieros, entre otros, como resultado de la ampliación de las TIC a todos los ámbitos de la universidad.

87 Tales como pago electrónico a proveedores, compras por la red, publicidad en medios electrónicos, envío de folletos electrónicos a los aspirantes de los programas remitidos a bases de datos especializadas (Mailing), espacios multimediales con transmisiones en directo y la venta de libros y revistas en línea y recaudos de inscripciones y matrículas en línea. En universidades a distancia y virtuales tienen un portafolio más amplio de servicios, como atención en línea, consejería virtual y chat financiero.

88 Universidad Nacional de Colombia. Acuerdo número 027 de 2005.

89 Fredy Yesid Mesa Jiménez, "Las tecnologías de la información y la comunicación en la universidad: Prospectiva 2.0", en Revista Pensamiento y Acción, vol. 10 No. 17 (2010): 105-110.

90 Recurso tecnopedagógico que permite anotar, dibujar y desarrollar una sesión didáctica con el apoyo de contenidos digitales interactivos.

91 Alonso García et al., La Pizarra Digital (España: Editor Cultiva libros, 2009), 9. 
en Colombia, según Smart en 2010, se han instalado más de 1.100 aulas digitales, (70\% en colegios públicos y el 30\% restante en colegios y universidades privadas $)^{92}$. Estos escenarios se deben visionar y seguir adaptando pedagógicamente al contexto, con el fin de no quedar rezagados.

\section{CONCLUSIÓN}

En Colombia, la empresa privada fue la pionera de la llegada de la computación, lo cual sucedió cuando en el contexto internacional esta disciplina tenía décadas de avances, siendo pocos los proyectos en el diseño y construcción, por ende desde sus inicios el país fue dependiente y consumidor de estas tecnologías. Los primeros computadores extendidos a la academia, impulsaron las primeras carreras y posgrados y al surgir los centros de cómputo, inicia la integración en algunas universidades desde finales de la década de los sesenta y en los setenta. Desde entonces, se ha invertido en infraestructura, cualificación ${ }^{93}$, integración pedagógica, entre otros, por medio de la autonomía universitaria.

El interés de las universidades por la integración transversal de sus procesos, originó que fuera uno de los actores de la conexión a Internet y de su propagación, así mismo, uno de los medios de investigación, actualización y generalización de la informática.

La integración de las TIC en las universidades fue diversa en estrategias, planes, políticas y en algunos casos, la improvisación; lo que generó desigualdades en la apropiación tecnológica y pedagógica, a pesar de la creciente y extensiva incorporación. El esfuerzo más amplio y visible se ha dado en las dependencias en educación virtual en instituciones presenciales y a distancia. Se han superado algunos obstáculos y se cuenta con lineamientos y políticas nacionales, lo cual había sido una omisión por décadas. Las TIC continúan con influencia en cada contexto y transforma roles, ambientes de aprendizaje y modelos pedagógicos.

92 Colombia Digital. "Colombia, en la era de las aulas digitales" (2010), http://www.colombiadigital.net/index.php?option=com_content $\&$ view $=$ article $\&$ id $=370:$ colombia-en-la-era-de-las-aulasdigitales\&catid=75:apropiacion-tic\&Itemid=274 (15 de agosto, 2010).

93 En ocasiones enfocada a un uso instrumental de la tecnología (el cual es necesario) y no pedagógico. 
Las tecnologías de la información y la comunicación en la universidad colombiana: evolución y prospectiva

En la última década se generalizó la modernización del sector educativo para cumplir con las funciones misionales, la articulación entre instituciones, optimizar costos y mejorar la competitividad y la productividad. Algunos programas, estudios y políticas, evidencian la intención de fortalecer la modalidad virtual, buscando mejorar los indicadores de cobertura, se está frente al aumento de la digitalización de los ejes misionales de la universidad y con una comunidad académica cada vez más a tono con su uso, pero se deben generar políticas y estrategias para continuar avanzando hacia la Web 2.0 y la filosofía Universidad 2.0.

Se requieren investigaciones más periódicas del avance de las TIC en la universidad colombiana, para valorar los efectos en la calidad e innovación educativa, además de estudios que recuperen la memoria histórica y el protagonismo de las universidades, para que las nuevas generaciones y la sociedad valoren sus aportes a la educación y al desarrollo del país.

\section{FUENTES}

Consejo Nacional de Política Económica y Social. Departamento Nacional de Planeación. Ministerio de Comunicaciones. Documento CONPES 3072 de 9 de febrero de 2000.

Colombia. Ley 115 de 1994.

Colombia. Presidencia de la República. Decreto 127 de 2001.

Colombia. Presidencia de la República. Decreto 3107 de 2003.

Consejo Nacional de Acreditación. El sistema de mejoramiento continuo del Consejo Nacional de Acreditación, (Bogotá: 2009). http://www.cna.gov.co/1741/articles-186379_recurso_1.pdf (22 de noviembre, 2011).

Departamento Administrativo Nacional de Estadística. Primer censo nacional de recursos informáticos en el sector público. Colombia: Ed. DANE, 1985.

Mejía Mazuera, Jaime. "Para qué sirven los computadores". Periódico El Tiempo, Bogotá, agosto 8 de 1990.

Entrevista a Vanegas Murcia, Eliécer. (Coordinación de planeación Gobierno en Línea), Bogotá, 6 de octubre de 2011.

Entrevista a Barbosa, Adriana (SISCONPES), Bogotá, 6 de octubre de 2011.

Entrevista a González, Rosario. (Dirección de infraestructura y energía sostenible. Departamento Nacional de Planeación), Bogotá, 6 de octubre de 2011.

Manjarrés, Carlos. "Presencia de Colombia en Internet", El Tiempo, Bogotá, 20 de noviembre, 1995. 
Universidad Nacional de Colombia. Acuerdo Número 027 de 2005.

Universidad Nacional de Colombia. Acuerdo Número 034 de 2004.

Universidad Pedagógica y Tecnológica de Colombia. Acuerdo No. 022 de 1994 del Consejo Superior Universitario.

Universidad Pedagógica y Tecnológica de Colombia. Resolución Rectoral Número 2848 de 2003.

Universidad Pedagógica y Tecnológica de Colombia. Resolución Rectoral Número 414 de 1972.

\section{REFERENCIAS}

Alonso García, Catalina; Alconada Fernández, Cristina; Gallego Gil, Domingo, y Dulac Ibergallartu, José. La Pizarra Digital. España: Editor Cultivalibros, 2009.

Ángel, Facundo. "La Educación Superior Virtual en Colombia", en La educación superior virtual en América Latina y el Caribe. ANUIES-UNESCO. México: ANUIES, 2004, 175-222.

Ávila Fernández, Alejandro; Calderón España, María Consolación; Corts Giner, María Isabel; Montero Pedrera, Ana María. Historia de la Educación, Cuestiones previas y perspectivas actuales. Sevilla: GI.P.E.S., 1996.

Barro Ameneiro, Senén y Bugarín Diz, Alberto. Fronteras de la computación. España: Ediciones Díaz de Santos, 2002.

Botero, Carolina. "Seminario del Ministerio de Educación en Colombia Estrategias para el Uso de Objetos de Aprendizaje y Redes de Alta Velocidad" (2006). http:/ /www.karisma.org.co/carobotero/ index.php/2006/07/27/seminario-del-ministerio-de-educacion-en-colombia-estrategiaspara-el-uso-de-objetos-de-aprendizaje-y-redes-de-alta-velocidad/.

Castaño Garrido, Carlos; Maiz Olazabalaga, Inmaculada; Garay Ruiz, Urtza; Bustillo Bayón, Jon; Tejada Garitano, Eneko. "Elearning 2.0", en Nuevos escenarios pedagógicos para el aprendizaje digital. Universidad del País Vasco (2009). http://ocw.ehu.es/ciencias-sociales-y-juridicas/ nuevos-escenarios-pedagogicos-para-el-aprendizaje-digital/bloque-3-estrategias-de-aprendizaje-2-0-para-la-innovacion-pedagogica/tema-1-elearning-2.0.

Coello Coello, Carlos. Breve historia de la computación y sus pioneros. México: Fondo de Cultura Económica, 2003.

Colombia Digital. "Colombia, en la era de las aulas digitales", (2010). http:/ /www.colombiadigital.net $/$ index.php?option $=$ com_content\&view $=$ article\&id $=370$ :colombia-en-la-era-de-lasaulas-digitales\&catid $=75$ :apropiacion-tic\&Itemid $=274$.

Correa, Leonardo. "La llamaron Clementina”, (2005) http://edant.clarin.com/suplementos/ informatica/2005/08/17/f-00511.htm.

El Tiempo, "Museo de Informática", Periódico El Tiempo, Bogotá, 8 de agosto de 1990. http:// www.eltiempo.com/archivo/documento/MAM-69828 

universidad colombiana: evolución y prospectiva

Finquelievich, Susana y Prince, Alejandro. Universidades y TICs en Argentina. Las universidades argentinas en la sociedad del conocimiento. 2005. http://www.scribd.com/doc/4940962/Finquelievich-y-PrinceLAS-UNIVERSIDADESARGENTINAS-EN-LA.

Forester, Tom. Sociedad de alta tecnología: la historia de la revolución de la tecnología de la información. México: Siglo Veintiuno Editores, 1992.

Guarín, Oscar. "ACIS, Memorias de la Informática en Colombia", Revista Sistemas. Memoria informática de Colombia. Edición $N^{\circ}$ 100. Asociación Colombiana de Ingenieros de Sistemas (2007). http://www.acis.org.co/fileadmin/Revista_100/ED_100_INVESTIGACION.pdf.

Hilbert, Martin, Sebastián, Bustos y João Carlos Ferraz. Estrategias nacionales para la sociedad de la información en América Latina y el Caribe. Naciones Unidas, 2005.

Jacovkis, Pablo. "Breve resumen de la bistoria de la computación en Argentina", (2004).

http:/ $/$ www.sadio.org.ar/ modules.php?op $=$ modload\&name $=$ News\&file $=$ article $\&$ sid $=50$.

Maldonado Granados, Luis Facundo y Maldonado Rey, Paola. "Nuevas tecnologías aplicadas a la educación. Estado del arte de la investigación, 1990-1999”, en Estados del Arte de la Investigación en Educación y Pedagogía en Colombia, editado por Myrian Henao Willes y Jorge Orlando Castro. Bogotá: ICFES, 2001, 146-147.

Maldonado Granados, Luis Facundo. "RIBIECOL - 20 años de vida", en Memorias Congreso Internacional de Informática Educativa. Aulas virtuales, diseño pedagógico, simulaciones y cibercultura, Popayán: 14, 15 y 16 de julio de 2010, CD-ROM. RIBIECOL.

Meneses, Arnaldo. El sector de las telecomunicaciones en Colombia en la década de los 90 's. Comisión de Regulación de Comunicaciones de Colombia, 2000. http://www.crcom.gov.co/images/ stories/crtdocuments/BibliotecaVirtual/publi_sector90/LibroTelec.pdf.

Mesa Jiménez, Fredy Yesid y Soler Fonseca, Ángela Marcela. "Políticas públicas en Tecnologías de la Información y la Comunicación y su incidencia en la universidad colombiana (2000-2008)", en Memorias Congreso de Investigación y Pedagogía II nacional I internacional. Perspectivas retos y transformaciones en contextos educativos. Maestría en Educación UPTC, 2011, 270-278.

Mesa Jiménez, Fredy Yesid. "Las tecnologías de la información y la comunicación en la universidad: Prospectiva 2.0”, en Revista Pensamiento y Acción. Vol. 10 N 17 (2010): 105-110.

Ministerio de Educación Nacional. Balance de la Revolución Educativa 2002-2010 (Bogotá: 2010). http://www.mineducacion.gov.co/1621/articles-231469_archivo_pdf_discurso.pdf.

Ministerio de Educación Nacional. Las tecnologías, aliadas estratégicas de la educación superior (Bogotá: 2006?). http://www.mineducacion.gov.co/cvn/1665/fo-article-103872.pdf.

Ministerio de Educación Nacional. "Mineducación lanzó campaña de educación superior virtual como una alternativa real". (2010). http://www.mineducacion.gov.co/cvn/1665/w3article-237475.html.

Ministerio de Educación Nacional. Uso de medios y tecnologías de información y comunicación en Educación Superior. (2007). http://www.colombiaaprende.edu.co/html/mediateca/1607/articles-126604_archivo.pdf.

Ministerio de Tecnologías de la Información y las Comunicaciones. "Vive Digital Colombia. Documento Vivo del Plan. Versión 1.0”. Bogotá: MEN, 2011. 
Montes, Álvaro. "Llegaron los computadores", 50 días que cambiaron la historia de Colombia”, en Revista Semana. (Bogotá: Editorial Planeta, 2004, 237-239.

Ortiz Arroyo, Daniel; Rodríguez Henríquez, Francisco; Coello Coello, Carlos. "Computadoras mexicanas: una breve reseña técnica e histórica". Revista Digital Universitaria. Universidad Nacional Autónoma de México, vol. 9, Nº 9 (2008). http://www.revista.unam.mx/vol.9/num9/ art63/art63.pdf

Pontificia Universidad Javeriana. Informe del Rector al Consejo de Regentes 2003. Bogotá: Editorial Pontificia Universidad Javeriana, 2003.

Rincón Cárdenas, Erick. Manual de derecho de comercio electrónico y de Internet. Bogotá: Editorial Universidad del Rosario, 2006.

Salcedo, Fernando. "Desde la academia, internet para Colombia". Revista de ingeniería Universidad de los Andes No 23 (2006): 146-148.

Tobón Lindo, Martha Isabel; Arbeláez Gómez, Martha Cecilia; Falcón Tomé, María del Carmen; Bedoya Sánchez, José Rubiel. La formación docente al incorporar las TIC en los procesos de enseñanza y aprendizaje. Una propuesta para la Universidad Tecnológica de Pereira. (2010) http://www. slideshare.net/misabell/el-docente-y-las-tic-4476500.

Universidad de los Andes. Sitio Web del Departamento de Ingeniería de Sistemas y Computación.http://sistemas.uniandes.edu.co/sitio/index.php?option $=$ com_content\&view $=$ articl e\&id=65\&Itemid $=90$.

Universidad de los Andes. Sitio Web del grupo de investigación LIDIE.http:/ / cife.uniandes. edu.co/web/index.php?option=com_content\&task=view\&id=209\&Itemid=519.

Uribe, Luis. "CODIDAC". Revista Tekhne de la Facultad de Ingeniería No 7. Universidad Católica Andrés Bello, 2004.

Uribe, Luis. Proyectos realizados. http://www.scribd.com/doc/20253455/UribeUSBProy1dPublicar.

Valencia Rincón, Juan Carlos. Internet en Colombia. Alucinación, información y comunicación, Tesis de maestría en Comunicación, Pontificia Universidad Javeriana, Facultad de Comunicación y Lenguaje, 1998.

Colombia Aprende ¿Qué es la educación virtual? (s.f.). http://www.colombiaaprende.edu.co/ $\mathrm{html} /$ productos/1685/article-229097.html.

Colombia Aprende. "Estrategia Planes TIC". (s.f.). http://www.colombiaaprende.edu.co/ html/directivos/1598/article-201182.html (20 de agosto, 2010).

Zapata Zapata, Donna. Contextualización de la enseñanza virtual en la educación superior. Bogotá: ICFES, 2002. http://blade1.uniquindio.edu.co/uniquindio/ntic/lineamientos/nuevos $\% 20$ libros/arc_88.pdf.

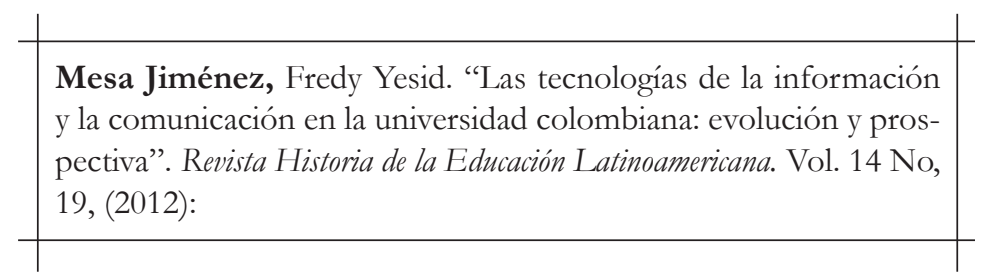

Rev. hist.edu.latinoam - Vol. 14 No. 19, julio-diciembre 2012 - ISSN: 0122-7238 - pp. 71 - 90 